\title{
Editorial: Therapy-induced metastasis
}

\author{
Olga A. Martin ${ }^{1} \cdot$ Robin L. Anderson ${ }^{2,3}$
}

Received: 11 June 2018 / Accepted: 18 June 2018 / Published online: 3 July 2018

(c) Springer Nature B.V. 2018

\begin{abstract}
The idea for this Special Issue originated from our recent review in Nature Reviews Clinical Oncology entitled "Does the mobilization of circulating tumour cells during cancer therapy cause metastasis?" Martin et al. (Nat Rev Clin Oncol 14:32-44, 2017). While preparing this review, it became evident that an overwhelming number of preclinical and clinical papers were implicating the involvement of all the major and indispensable cancer treatment modalities in causing increased numbers of tumour cells in circulation (CTCs), and potentially increased risk of distant metastasis. This led to our decision to expand the topic by addressing some of the issues associated with therapy-induced tumour progression. Here, we present papers from ten research groups who give a comprehensive coverage of the biological processes and clinical procedures that can lead to enhanced metastasis and/or tumour recurrence. Our authors provide evidence that all the common therapies, including radiotherapy, chemotherapy, fine needle biopsies, surgical procedures and anaesthesia have the potential to contribute to tumour progression.
\end{abstract}

Keywords Cancer therapy $\cdot$ Radiotherapy $\cdot$ Chemotherapy $\cdot$ Surgery $\cdot$ Metastasis

\section{Radiotherapy}

Although the idea that radiotherapy contributes to metastasis was discussed since as early as 1949 [1], and potential mechanisms were suggested in 1991 by von Essen [2], the review presented here by Blyth et al. [3] updates the evidence collected over the past few decades. Dramatic progress in radiotherapy technology has significantly improved efficacy and accuracy of radiation treatments. Similarly, new methodologies in molecular and cell biology have permitted further investigation of cellular radiation responses, as well as responses of whole organisms. There is now evidence of tumour cells escaping from an irradiated tumour during treatment via the venous system or the lymphatics, and that acquired properties of these irradiated cells can determine their viability, aggressiveness, and metastatic capacity [4, 5]. Tumour cells can enter the bloodstream as single cells

Robin L. Anderson

robin.anderson@onjcri.org.au

Peter MacCallum Cancer Centre, Melbourne, VIC, Australia

2 Olivia Newton-John Cancer Research Institute, 145 Studley Road, Heidelberg, VIC, Australia

3 La Trobe University School of Cancer Medicine, Bundoora, VIC, Australia or as clusters. Clustering of CTCs enhances their survival, adhesion, local invasion, angiogenesis and intravasation. In fact, it has been reported that CTC clusters are more likely to form metastases and be associated with bad prognosis [6]. CTCs are bound together in a cluster by fibrin and covered by platelets, and such clusters, as discussed in this issue by Burbury and MacManus [7] can add to cancer-induced haemostatic dysfunction, such as hypercoagulation. There are other contributors to enhanced coagulation, including expression of tissue factor on the surface of tumour cells, secretion of cancer procoagulant and other cysteine proteinases that release highly procoagulating microparticles, elevation of plasma concentration of pro-inflammatory and pro-angiogenic cytokines and growth factors, and deregulation of activation and inhibition of fibrinolysis [7].

In a previous report, Vilalta et al. reported that in preclinical models, irradiation of tumours leads to tumour cell migration from, and back to the treated tumour, providing a mechanism for tumour recurrence [8]. The process is regulated by the cytokine, granulocyte-macrophage colony stimulating factor (GM-CSF), produced by irradiated tumour cells. GM-CSF is currently employed in clinical practice to treat neutropenia in cancer patients. In their new study in this special issue, Vilalta et al. show that intravenously injected cells can home to both irradiated and un-irradiated sites [9], implicating a systemic factor. However, systemic delivery of 
GM-CSF could not replicate the migratory effects triggered by an irradiated tumour, hence the precise contribution of GM-CSF to migration remains to be established.

\section{Chemotherapy}

Granulocyte-colony stimulating factor, G-CSF, is also used extensively as a support therapy for patients undergoing chemotherapy, to avoid the complications of neutropenia. In addition, G-CSF is used to assist in haematopoietic stem cell mobilization prior to autologous bone marrow transplants. However, G-CSF has a "dark side". In this issue, Yeo et al. [10] review the literature reporting the mobilization of tumour-associated neutrophils that promote tumour dissemination and metastasis in preclinical models. They also summarise studies reporting human tumours where G-CSF or its receptor is expressed, raising the question of whether G-CSF promotes the growth of some human tumours. However, upon review of the available literature, they conclude that so far, there is no evidence for adverse effects, in terms of tumour promotion, from administering G-CSF to cancer patients.

Karagiannis et al. [11] reported recently that chemotherapy for breast cancer, while decreasing tumour size, in some cases can promote metastatic dissemination. The increased metastasis was associated with increased density of structures called the tumour microenvironment of metastasis (TMEM). The TMEM is composed of tumour cells, Tie2 positive macrophages and vascular endothelial cells in direct contact [11]. Here, they follow up with a comprehensive review of the metastatic process and potential mechanisms of chemotherapyinduced metastasis [12]. They suggest that the host response to tissue damage caused by chemotherapy is to initiate a tissue repair program involving mobilization of bone marrow progenitors that are subsequently exploited by the tumour cells to promote metastasis. Metastasis can also be achieved through enhanced epithelial-to-mesenchymal transition (EMT), an increased pro-inflammatory response, acquired drug resistance, and through the pro-metastatic properties of CTCs. They follow-up with some suggestions of how the pro-metastatic aspects of chemotherapy can be restricted [12].

The challenge of cancer treatment-induced EMT and drug resistance has been further developed in this special issue. Redfern et al. [13] review EMT changes in cancer by analogy with "The Kraken Wakes". Taking the name from an Alfred Lord Tennyson sonnet, the John Wyndham novel with this name describes the invasion of Earth by unseen aliens. Like these aliens, tumour cells find a way to invade through EMT, triggering treatment insensitivity, aggressiveness, invasion and metastasis. EMT is a broad mechanism of resistance to cancer treatment that can be triggered by a wide spectrum of cytotoxic chemotherapies, targeted therapies and radiotherapy. The authors review studies where the expression of EMT markers in post-therapy biopsies correlates with reduced treatment benefits and an overall poor prognosis. Conversely, they list the benefit of chemotherapies that trigger a reversal of EMT, resulting in mesenchymal to epithelial transition (MET) and an increased overall survival of patients. Redfern et al. also review therapeutic approaches to manipulate EMT to the benefit of the patient [13]. The review by Nikolaou et al. [14] addresses the mechanisms of resistance to cancer drugs, leading to repopulation within a treated tumour, or systemic dissemination of resistant tumour cells. They suggest that acquired drug resistance can be achieved through either genetic or epigenetic mechanisms, whereas intrinsic drug resistance is often mediated through multi-drug resistance at the level of membrane transporter proteins or altered cellular metabolic processes. Involvement of other determinants of the cellular drug response, such as DNA repair, tumour microenvironment and properties of cancer stem cells adds to the complexity of this clinical problem [14].

\section{Surgery}

Surgical procedures that are implicated in the promotion of metastasis are addressed in reviews from Alieva et al. [15] and Behrenbruch et al. [16]. Using high-resolution intra-vital microscopy in a mouse glioblastoma model, Alieva et al. demonstrated previously that even a minor surgical trauma, such as fine-needle biopsy, could induce tumour cell migration and proliferation that could be prevented by blocking macrophage recruitment to the site of injury through inhibition of chemokine (C-C motif) ligand 2 (CCL-2) [17]. In this issue, Alieva et al. [15] further explore the negative side-effects of tumour resection and biopsies, which are indispensable curative and diagnostic procedures. Surgery can physically disrupt tumours and surrounding normal tissues and blood vessels, leading to migration of residual tumour cells. Surgery can also induce a wound healing response that leads to profound local and systemic effects, such as immunosuppression and activation of coagulation, further impairing anti-tumour immunity and favouring tumour cell survival. Behrenbruch et al. [16] review the literature regarding the effects of surgery on progression of colorectal cancer, implicating tumour cell release into circulation and suppression of anti-tumour immunity. They review the data demonstrating that catecholamines and prostaglandins are major contributors to the surgical stress response and how these factors stimulate tumour cell proliferation through activation of the beta-adrenoreceptor or Cox 2 on the tumour cells. They cite studies reporting that peri-operative use of inhibitors of the beta-adrenoreceptor or of Cox 2 reduces biomarkers associated with metastasis. The Hollande research group is known for their studies on the heterogeneity of colorectal 
cancer $[18,19]$. In this paper, they report experiments in which they transduced patient-derived colorectal cancer cells with Lentiviral Gene Ontology (LeGo) vectors encoding red, green and blue (RGB) fluorescent proteins, resulting in cell subpopulations with individual stable colour "signatures". By tracking these subpopulations after tumour cell inoculation into mice and treatment with a beta-adrenoreceptor agonist, they show that the individual cells have differing stress responses, thereby demonstrating the heterogeneity of the patient's tumour. The LeGo/ RGB technology is a promising tool for revealing and understanding tumour cell heterogeneity [16].

Anesthesia is a critical aspect of surgical oncology, with a majority of cancer patients receiving anesthesia during their surgery, diagnostic or palliative procedures. Dubowitz et al. [20] in this issue review the current evidence for anaesthetic agents in the modulation of cancer progression. They report that different anaesthetic agents and techniques are likely to have differing consequences for long-term outcome cancer patients subjected to surgery. Both the type (volatile or intravenous anesthesia) and technique (neuraxial or general anesthesia) can affect the response of tumour cells and immune cells. While a lot has been learnt, many questions still remain, especially related to the mechanisms and timing of systemic effects of surgery and anesthesia.

\section{Conclusion: identification of prevention or intervention strategies}

The challenge remains to weigh up benefit versus detriment of therapy. The clinical modalities described here are essential for cancer control, but unfortunately they may have some negative consequences in some patients. Further understanding of how these modalities are able to assist in tumour cell escape and metastasis will enable strategies to minimize these negative side-effects. For example, Song et al. [21] investigated whether the number of CTCs in patients undergoing surgery for NSCLC was affected by the sequence of events in ligating the pulmonary artery and pulmonary vein. They found that ligation of the pulmonary vein prior to ligation of the pulmonary artery reduced signals associated with tumour cells released into circulation. With sufficient knowledge of mechanisms of therapy-induced metastasis, other such interventions will be developed to minimize unwanted side-effects and improve outcome for cancer patients.

\section{References}

1. Kaplan HS, Murphy ED (1949) The effect of local roentgen irradiation on the biological behavior of a transplantable mouse carcinoma; increased frequency of pulmonary metastasis. J Natl Cancer Inst 9(5-6):407-413

2. von Essen CF (1991) Radiation enhancement of metastasis: a review. Clin Exp Metastasis 9(2):77-104

3. Blyth BJ et al (2017) Radiation therapy-induced metastasis: radiobiology and clinical implications. Clin Exp Metastasis. https://doi. org/10.1007/s10585-017-9867-5

4. Martin OA et al (2014) Mobilization of viable tumor cells into the circulation during radiation therapy. Int J Radiat Oncol Biol Phys 88(2):395-403

5. Errico A, Radiotherapy (2014) A double-edged sword for NSCLC? Nat Rev Clin Oncol 11(2):66

6. Aceto $\mathrm{N}$ et al (2014) Circulating tumor cell clusters are oligoclonal precursors of breast cancer metastasis. Cell 158(5):1110-1122

7. Burbury K, MacManus M (2018) The coagulome and the oncomir: impact of cancer-associated haemostatic dysregulation on the risk of metastasis. Clin Exp Metastasis. https://doi.org/10.1007/s1058 5-018-9875-0

8. Vilalta M et al (2014) Recruitment of circulating breast cancer cells is stimulated by radiotherapy. Cell Rep 8(2):402-409

9. Vilalta $\mathrm{M}$ et al (2018) The role of granulocyte macrophage colony stimulating factor (GM-CSF) in radiation-induced tumor cell migration. Clin Exp Metastasis. https://doi.org/10.1007/s1058 5-018-9877-y

10. Yeo B et al (2018) The dark side of granulocyte-colony stimulating factor-a supportive therapy with potential to promote tumour progression. Clin Exp Metastasis. https://doi.org/10.1007/s1058 5-018-9917-7

11. Karagiannis GS et al (2017) Neoadjuvant chemotherapy induces breast cancer metastasis through a TMEM-mediated mechanism. Sci Transl Med. 9(397):eaan0026

12. Karagiannis G, Condeelis J, Oktay M (2018) Chemotherapyinduced metastasis: mechanisms and translational opportunities. Clin Exp Metastasis. https://doi.org/10.1007/s10585-017-9870-x

13. Redfern AD, Spalding LJ, Thompson EW (2018) The Kraken wakes: induced EMT as a driver of tumour aggression and poor outcome. Clin Exp Metastasis. https://doi.org/10.1007/s10585-018-9906-x

14. Nikolaou $\mathrm{M}$ et al (2018) The challenge of resistance in cancer treatment: a current overview. Clin Exp Metastasis. https://doi. org/10.1007/s10585-018-9903-0

15. Alieva M, van Rhennen J, Broekman M (2018) Potential impact of invasive surgical procedures on primary tumor growth and metastasis. Clin Exp Metastasis. https://doi.org/10.1007/s1058 5-018-9896-8

16. Behrenbruch $C$ et al. (2018) Surgical stress response and promotion of metastasis in colorectal cancer: a complex and heterogeneous process. Clin Exp Metastasis. https://doi.org/10.1007/s1058 5-018-9873-2

17. Alieva $M$ et al (2017) Preventing inflammation inhibits biopsymediated changes in tumor cell behavior. Sci Rep 7(1):7529

18. Grillet $\mathrm{F}$ et al (2017) Circulating tumour cells from patients with colorectal cancer have cancer stem cell hallmarks in ex vivo culture. Gut 66(10):1802-1810

19. Paquet-Fifield S et al (2018) Tight junction protein claudin-2 promotes self-renewal of human colorectal cancer stem-like cells. Cancer Res 78(11):2925-2938

20. Dubowitz J, Sloan E, Riedel B (2018) Implicating anaesthesia and the perioperative period in cancer recurrence and metastasis. Clin Exp Metastasis. https://doi.org/10.1007/s10585-017-9862-x

21. Song PP et al (2013) Effects of different sequences of pulmonary artery and vein ligations during pulmonary lobectomy on blood micrometastasis of non-small cell lung cancer. Oncol Lett 5(2):463-468

22. Martin OA et al (2017) Does the mobilization of circulating tumour cells during cancer therapy cause metastasis? Nat Rev Clin Oncol 14(1):32-44 